\title{
Combined Measurement of Inclusive ep Scattering Cross Sections at HERA
}

\author{
Oleksii Turkot* on behalf of the $\mathrm{H} 1$ and ZEUS collaborations \\ DESY \\ E-mail: eleksii.turkotedesy.de
}

All previously published measurements of inclusive deep inelastic scattering (DIS) by the H1 and ZEUS collaborations at HERA have been combined for neutral and charged current unpolarised $\mathrm{e}^{ \pm} \mathrm{p}$ cross sections. The data were taken at electron beam energy of $27.5 \mathrm{GeV}$ and proton beam energies of $920,820,575$ and $460 \mathrm{GeV}$. The combined data correspond to a luminosity of about $1 \mathrm{fb}^{-1}$ and span six orders of magnitude in negative four-momentum-transfer squared, $Q^{2}$, and Bjorken $x$. The correlations of systematic uncertainties were taken into account, resulting in significantly improved precision.

The European Physical Society Conference on High Energy Physics 22-29 July 2015

Vienna, Austria

${ }^{*}$ Speaker. 


\section{Introduction}

Measurements of deep inelastic scattering (DIS) of electrons ${ }^{1}$ on protons at HERA provide an important input for determination of proton structure and quark-gluon dynamics as described by perturbative Quantum Chromodynamics (pQCD). For most of the data taking periods HERA collider operated with centre-of-mass energy of up to $\sqrt{s}=319 \mathrm{GeV}$. This enabled the two collaborations, $\mathrm{H} 1$ and ZEUS, to explore a large phase space in Bjorken $x, x_{B j}$, and negative fourmomentum-transfer squared, $Q^{2}$. The kinematic range of measured neutral current (NC) cross sections was $0.045 \leq Q^{2} \leq 50000 \mathrm{GeV}^{2}$ and $6 \cdot 10^{-7} \leq x_{B j} \leq 0.65$, at values of the inelasticity, $y=Q^{2} /(s x)$, of $0.005 \leq y \leq 0.95$. Cross sections for charged current (CC) interactions were measured for $200 \leq Q^{2} \leq 50000 \mathrm{GeV}^{2}$ and $1.3 \cdot 10^{-2} \leq x_{B j} \leq 0.40$, at values of the inelasticity of $0.037 \leq y \leq 0.76$.

HERA collider was operated in two phases: HERA I, during 1992-2000, and HERA II, during 2002-2007. All data used in combination were taken at electron beam energy of $27.5 \mathrm{GeV}$ and four different proton beam energies of $E_{p} \simeq 920,820,575$ and $460 \mathrm{GeV}$ resulting in center-of-mass energies of $\sqrt{s}=318,300,251$ and $225 \mathrm{GeV}$. During the HERA I period, each experiment collected about $100 \mathrm{pb}^{-1}$ of $\mathrm{e}^{+} \mathrm{p}$ and $15 \mathrm{pb}^{-1}$ of $\mathrm{e}^{-} \mathrm{p}$ data. These data were the basis of a combination published previously [1]. During the HERA II period, each experiment added about $150 \mathrm{pb}^{-1} \mathrm{of} \mathrm{e}^{+} \mathrm{p}$ and $235 \mathrm{pb}^{-1}$ of $\mathrm{e}^{-} \mathrm{p}$ data. As a result, both experiments, H1 and ZEUS, collected approximately $0.5 \mathrm{fb}^{-1}$ of luminosity, divided almost equally between $\mathrm{e}^{+} \mathrm{p}$ and $\mathrm{e}^{-} \mathrm{p}$ scattering. The combination presented now [2] includes all published H1 [3-10] and ZEUS [11-24] measurements from HERA I and HERA II on inclusive DIS in NC and CC reactions.

\section{Combination of the Measurements}

The data were taken with several centre-of-mass energies $\sqrt{s}$ and the double-differential cross sections were published by the H1 and ZEUS collaborations for different reference $\left(Q^{2}, x_{B j}\right)$ grids. In order to average a set of data points, at first they had to be translated to a common $\sqrt{s}_{\text {com }}$ and $\left(Q_{\text {grid }}^{2}, x_{B j, g r i d}\right)$ grid. Three common centre-of-mass values, $\sqrt{s}_{\text {com }, i}$, with $\sqrt{s}_{\text {com }, 1}=318 \mathrm{GeV}$ $\left(E_{p}=920 \mathrm{GeV}\right.$ and $\left.E_{p}=820 \mathrm{GeV}\right), \sqrt{s}_{\text {com }, 2}=252 \mathrm{GeV}\left(E_{p}=575 \mathrm{GeV}\right)$, and $\sqrt{s}_{\text {com }, 3}=225 \mathrm{GeV}$ $\left(E_{p}=460 \mathrm{GeV}\right)$ were chosen for data combination. A part of $E_{p}=820 \mathrm{GeV}$ data with $y \geq 0.35$ was kept at $\sqrt{s}=301 \mathrm{GeV}$. Two common $\left(Q_{\text {grid }}^{2}, x_{B j, \text { grid }}\right)$ grids were used, one for $\sqrt{s}_{\text {com }, 1}$ and another one for $\sqrt{s}_{\text {com }, 2}$ and $\sqrt{s}_{\text {com,3 }}$. The grids are depicted in Fig. 1 (left). They have a different structure in $y$ such that the corrections due to translation were minimised. The translation scale factors, SF, were calculated as ratios of the predicted double differential cross sections in the actual $\sqrt{s},\left(Q^{2}, x_{B j}\right)$ and the common $\sqrt{s}_{\text {com }, i},\left(Q_{\text {grid }}^{2}, x_{B j, g r i d}\right)$ grids. The predictions were obtained by performing the fits to the data with HERAFitter tool [25]. For $Q^{2} \geq 3 \mathrm{GeV}^{2}$, a QCD fit within the DGLAP formalism was performed, and for $Q^{2} \leq 4.9 \mathrm{GeV}^{2}$ a fit using the fractal model $[3,27]$ was obtained. For overlaping region $3 \mathrm{GeV}^{2} \leq Q^{2} \leq 4.9 \mathrm{GeV}^{2}$, the linear average of the scale factors was used.

Next data points were averaged within common grid points. For this, the HERAverager [26] tool was used. It uses a $\chi^{2}$-minimisation method, based on the assumption that there is only one

\footnotetext{
${ }^{1}$ Here and afterwards "electron" refers to both electrons and positrons, unless otherwise stated.
} 

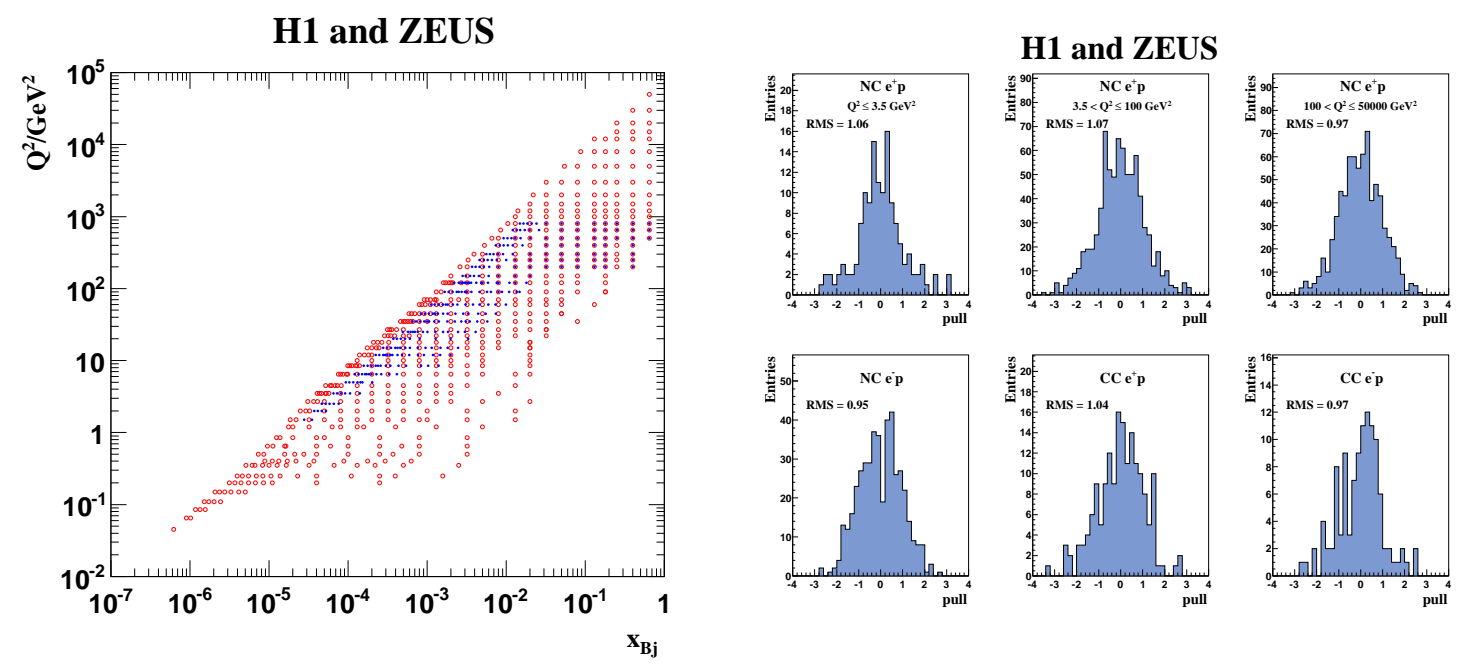

Figure 1: The points of two grids (left) used for the combination: grid 1 (open circles) was used for data with proton beam energies of 920 and $820 \mathrm{GeV}$, grid 2 (dots) was used for data with proton beam energies of 575 and $460 \mathrm{GeV}$, and the distribution of pulls $p$ (right) for $\mathrm{NC}$ and $\mathrm{CC} \mathrm{e}^{ \pm} \mathrm{p}$ data samples: there are no entries outside the histogram ranges and RMS gives the root mean square of each distribution.

correct value for the cross section for each process at each point of the phase space, described in detail in [3]. The used $\chi^{2}$ function takes into account the correlated and uncorrelated systematic uncertainties and allows for shifts of the data to accommodate the correlated uncertainties. For a single data set, $d s$, the $\chi^{2}$ function is defined as

$$
\chi_{\text {exp }, d s}^{2}(\mathbf{m}, \mathbf{b})=\sum_{i, d s}+\sum_{j, b}=\sum_{i} \frac{\left[m^{i}-\sum_{j} \gamma_{j}^{i} m^{i} b_{j}-\mu^{i}\right]^{2}}{\delta_{i, s t a t}^{2} \mu^{i}\left(m^{i}-\sum_{j} \gamma_{j}^{i} m^{i} b_{j}\right)+\left(\delta_{i, u n c o r r} m^{i}\right)^{2}}+\sum_{j} b_{j}^{2},
$$

where $\mathbf{m}$ is the vector of averaged, or "true" values of cross sections, $\mathbf{b}$ represents the shifts with respect to the correlated systematic uncertainties, $\mu^{i}$ is the measured value at a point $i$, and $\gamma_{j}^{i}$, $\delta_{i, s t a t}$ and $\delta_{i, \text { uncorr }}$ are the relative correlated systematic, relative statistical and relative uncorrelated systematic uncertainties, respectively. For measured points, $k$, contributing to averaged point $i$ of $\left(Q_{\text {grid }}^{2}, x_{\text {grid }}\right)$ grid, pulls $p^{i, k}$ were defined as $p^{i, k}=\frac{\mu^{i, k}-\mu^{i, \text { ave }}\left(1-\sum_{j} \gamma_{j}^{i, k} b_{j, a v e}\right)}{\sqrt{\Delta_{i, k}^{2}-\Delta_{i, \text { ave }}^{2}}}$, where $\Delta_{i, k}$ and $\Delta_{i, \text { ave }}$ are the statistical and uncorrelated systematic uncertainties added in a quadrature for the point $k$ and the average, respectively. The distribution of pulls shows no tensions for all processes, as can be seen in Fig. 1 (right).

Finally, procedural uncertainties were evaluated. They are introduced by the choices made for the combination and take into account the possible influence of different evaluations of relative systematic uncertainties, possible correlations between the H1 and ZEUS estimations of the photoproduction background and hadronic energy scales and treatment of large pulls of systematic uncertainties. The typical values of procedural uncertainties are below $1 \%$, reaching the fewpercents level for low- $Q^{2}$, high- $Q^{2}$ and reduced proton beam energy data. 


\section{Results}

In total, 2927 data points corresponding to an integrated luminosity of about $1 \mathrm{fb}^{-1}$ were combined into one coherent data set of 1307 cross-section values. The combination showed a good consistency of separate data sets with $\chi^{2} / n_{d o f}=1685 / 1620$. In Fig. 目 (left), the individual and the combined reduced cross sections for $\mathrm{NC} \mathrm{e}^{-} \mathrm{p}$ DIS scattering are shown as a function of $Q^{2}$ for selected $x_{B j}$ values, and in Fig. 目 (right) the inclusive $\mathrm{NC}^{-} \mathrm{p}$ and $\mathrm{e}^{+} \mathrm{p}$ combined HERA data together with fixed-target data are compared to HERAPDF2.0 NLO. The combined CC $\mathrm{e}^{-} \mathrm{p}$ cross sections as a function of $x_{B j}$ for 10 different values of $Q^{2}$ are shown in Fig. 3 (left), and Fig. 3 (right) present the cross sections for CC inclusive $\mathrm{e}^{-} \mathrm{p}$ and $\mathrm{e}^{+} \mathrm{p}$ scattering together with HERAPDF2.0 NLO predictions. The results of the combination of the data with lower proton beam energies are shown in Fig. 4 . The improvement due to combination is clearly visible on all plots.
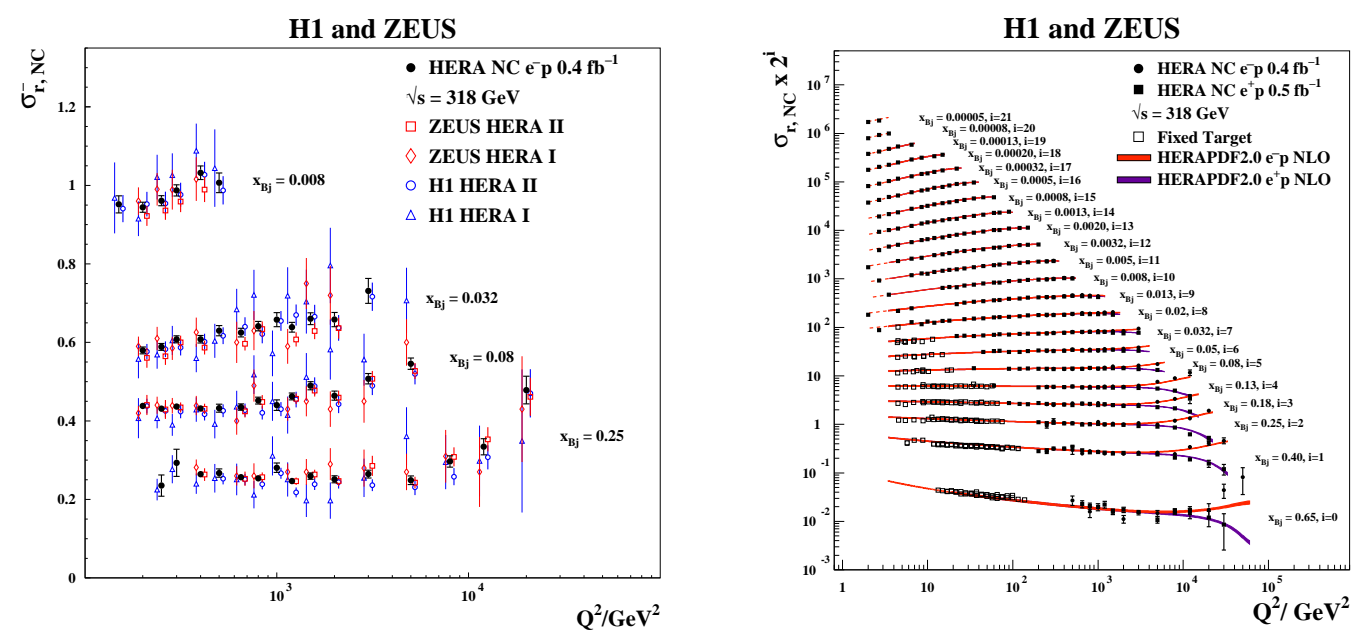

Figure 2: The combined HERA inclusive $\mathrm{NC}^{-} \mathrm{p}$ reduced cross sections as a function of $Q^{2}$ for six selected $x_{B j}$ bins compared to the individual $\mathrm{H} 1$ and ZEUS data (left) and inclusive $\mathrm{NC}^{-} \mathrm{p}$ and $\mathrm{e}^{+} \mathrm{p}$ reduced cross sections together with fixed-target data and the predictions of HERAPDF2.0 NLO (right). Errors bars represent the total uncertainties of the data and bands represent the total uncertainties on the predictions.

\section{Summary}

Final measurements of inclusive deep inelastic scattering by the H1 and ZEUS collaborations have been combined for neutral and charged current unpolarised $\mathrm{e}^{ \pm} \mathrm{p}$ cross sections. The data from many measurements made independently by the two experiments proved to be consistent with $\chi^{2} / n_{\text {dof }}=1685 / 1620$. The combined HERA inclusive DIS cross sections correspond to an integrated luminosity of about $1 \mathrm{fb}^{-1}$ and form a coherent data set spanning six orders of magnitude, both in $Q^{2}$ and $x_{B j}$. All correlations of systematic uncertainties were taken into account, resulting in their significant reduction. Presented combined cross sections are the most precise measurement ever published for $\mathrm{e}^{+} \mathrm{p}$ scattering over such a large kinematic range. They are a major legacy of HERA. 

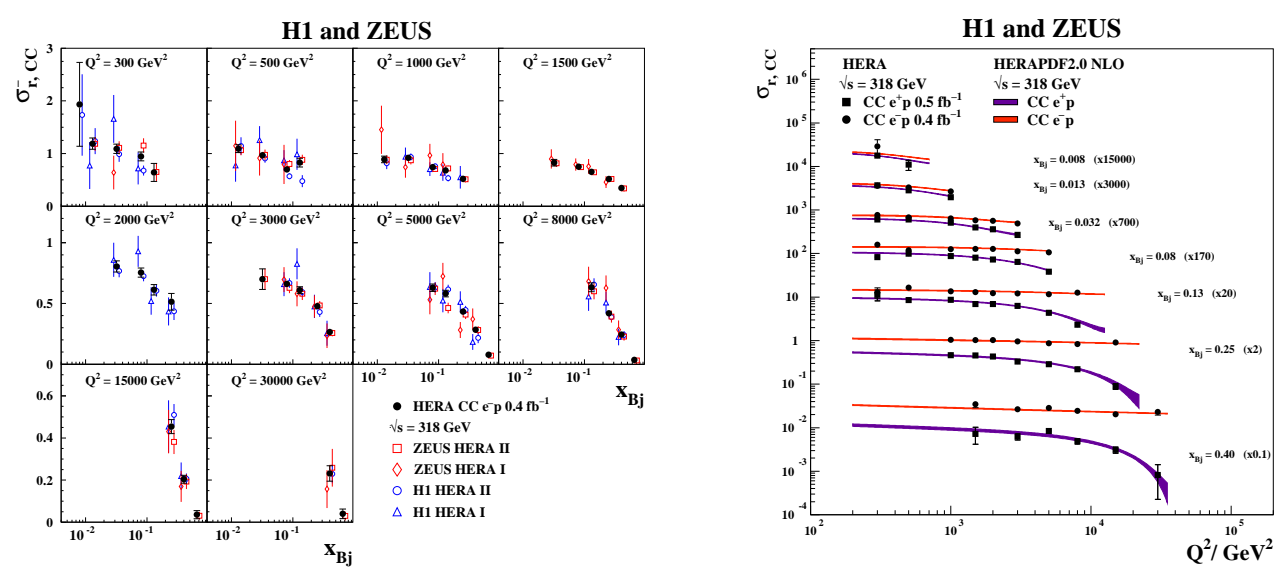

Figure 3: The combined HERA inclusive $\mathrm{CC}^{-}$p reduced cross sections as a function of $x_{B j}$ for 10 different values of $Q^{2}$ compared to the individual H1 and ZEUS data (left) and inclusive $C C \mathrm{e}^{-} \mathrm{p}$ and $\mathrm{e}^{+} \mathrm{p}$ reduced cross sections together with the predictions of HERAPDF2.0 NLO (right). Errors bars represent the total uncertainties of the data and bands represent the total uncertainties on the predictions.
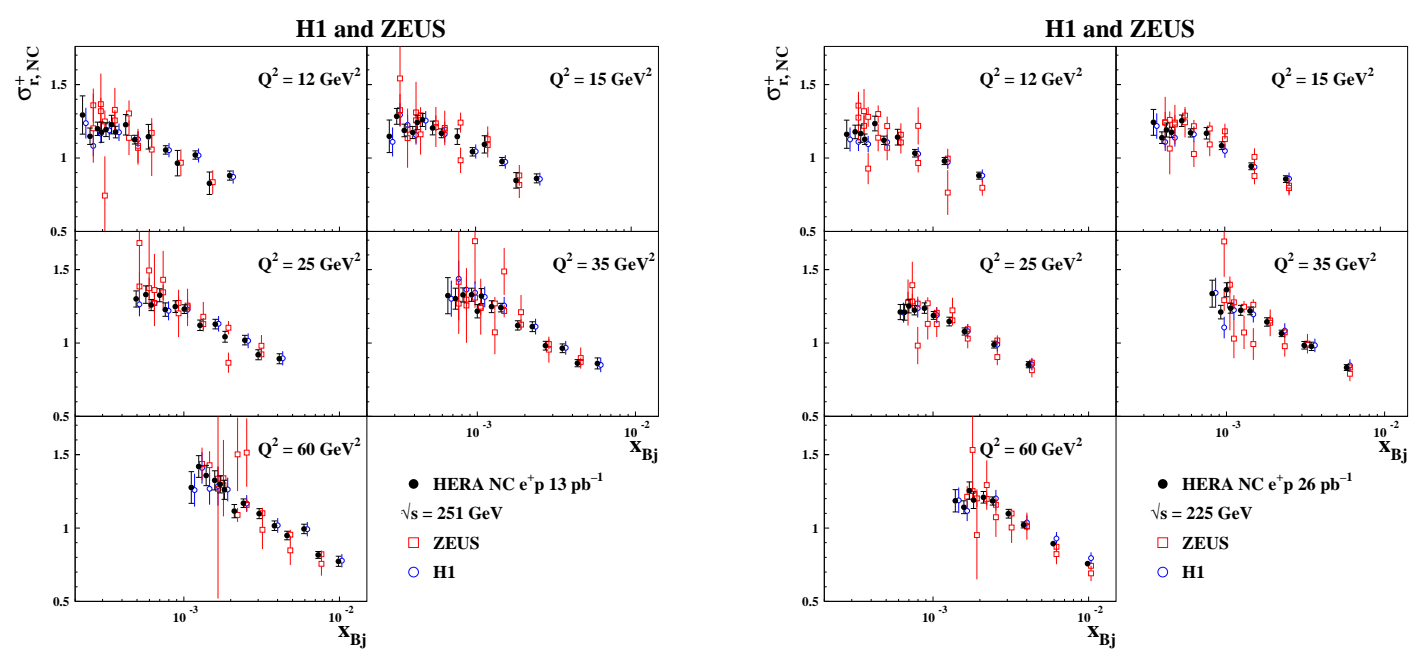

Figure 4: The combined HERA data for the inclusive $\mathrm{NC} \mathrm{e}^{+} \mathrm{p}$ reduced cross section at $E_{p}=575 \mathrm{GeV}$ (left) and at $E_{p}=460 \mathrm{GeV}$ (right) as a function of $x_{B j}$ for five different values of $Q^{2}$ compared to the individual $\mathrm{H} 1$ and ZEUS data which were the input to the averaging procedure. The individual measurements are displaced horizontally for better visibility. Errors bars represent the total uncertainties. 


\section{References}

[1] H1 and ZEUS Collaboration, JHEP 1001 (2010) 109 [arXiv: 0911.0884 ].

[2] H1 and ZEUS Collaboration, submitted to EPJC (2015) [arXiv:1506.06042v1].

[3] H1 Collaboration, Eur. Phys. J. C63 (2009) 625 [arXiv: 0904 . 0929].

[4] H1 Collaboration, Eur. Phys. J. C64 (2009) 561 [arXiv : 0904 . 3513].

[5] H1 Collaboration, Eur. Phys. J. C13 (2000) 609 [hep-ex/ 9908059 ].

[6] H1 Collaboration, Eur. Phys. J. C19 (2001) 269 [hep-ex/ 0012052 ].

[7] H1 Collaboration, Eur. Phys. J. C30 (2003) 1 [hep-ex/ 0304003 ].

[8] H1 Collaboration, JHEP 1209 (2012) 061 [arXiv: 1206 . 7007].

[9] H1 Collaboration, Eur. Phys. J. C74 (2014) 2814 [arXiv: 1312 . 4821].

[10] H1 Collaboration, Eur. Phys. J. C71 (2011) 1579 [arXiv: 1012 . 4355].

[11] ZEUS Collaboration, Phys. Lett. B407 (1997) 432 [hep-ex/ 9707025$].$

[12] ZEUS Collaboration, Phys. Lett. B487 (2000) 53 [hep-ex/ 0005018 ].

[13] ZEUS Collaboration, Eur. Phys. J. C7 (1999) 609 [hep-ex/9809005].

[14] ZEUS Collaboration, Eur. Phys. J. C21 (2001) 443 [hep-ex/0105090].

[15] ZEUS Collaboration, Eur. Phys. J. C12 (2000) 411, [Erratum-ibid. C27 (2003) 305], [hep-ex/9907010].

[16] ZEUS Collaboration, Eur. Phys. J. C28 (2003) 175 [hep-ex/0208040].

[17] ZEUS Collaboration, Phys. Lett. B539 (2002) 197, [Erratum-ibid. B552 (2003) 308], [hep-ex/0205091].

[18] ZEUS Collaboration, Phys. Rev. D70 (2004) 052001 [hep-ex / 0401003 ].

[19] ZEUS Collaboration, Eur. Phys. J. C32 (2003) 1 [hep-ex/ 0307043$].$

[20] ZEUS Collaboration, Eur. Phys. J. C62 (2009) 625 [arXiv: 0901 . 2385].

[21] ZEUS Collaboration, Eur. Phys. J. C61 (2009) 223 [arXiv: 0812 . 4620].

[22] ZEUS Collaboration, Phys. Rev. D87 (2013) 052014 [arXiv:1208.6138].

[23] ZEUS Collaboration, Eur. Phys. J. C70 (2010) 945 [arXiv: 1008 . 3493].

[24] ZEUS Collaboration, to be published in Phys. Lett. B (2014) [arXiv: 1404 . 6376].

[25] https://www.herafitter.org/HERAFitter/

[26] https://wiki-zeuthen.desy.de/HERAverager/

[27] T. Lastovicka, Eur. Phys. J. C24 (2002) 529 [hep-ph / 0203260$].$ 